\title{
Remote cardiac rehabilitation is a good alternative of outpatient cardiac rehabilitation in the COVID-19 era
}

\author{
Atsuko Nakayama ${ }^{*}$, Naoko Takayama², Momoko Kobayashi², Kanako Hyodo², Naomi Maeshima², \\ Fujiwara Takayuki ${ }^{1}$, Hiroyuki Morita ${ }^{1 *}$ and Issei Komuro ${ }^{1}$
}

\begin{abstract}
Background: In the wake of the coronavirus disease 2019 (COVID-19) pandemic, people need to practice social distancing in order to protect themselves from SARS-CoV-2 infection. In such stressful situations, remote cardiac rehabilitation (CR) might be a viable alternative to the outpatient CR program.

Methods: We prospectively investigated patients hospitalized for heart failure (HF) with a left ventricular ejection fraction of $<50 \%$. As for patients who participated in the remote CR program, telephone support was provided by cardiologists and nurses who specialized in HF every 2 weeks after discharge. The emergency readmission rate within 30 days of discharge was compared among the outpatient $C R$, remote $C R$, and non-CR groups, and the EQ$5 \mathrm{D}$ score was compared between the outpatient $C R$ and remote $C R$ groups.

Results: The participation rate of HF patients in our remote CR program elevated during the COVID-19 pandemic. As observed in the outpatient CR group $(n=69)$, the emergency readmission rate within 30 days of discharge was lower in the remote $\mathrm{CR}$ group $(n=30)$ than in the non-CR group $(n=137)(P=0.02)$. The EQ-5D score was higher in the remote $C R$ group than in the outpatient $C R$ group $(P=0.03) 30$ days after discharge.

Conclusions: Remote $C R$ is as effective as outpatient $C R$ for improving the short-term prognosis of patients hospitalized for heart failure post-discharge. This suggests that the remote CR program can be provided as a good alternative to the outpatient CR program.
\end{abstract}

Keywords: Cardiac rehabilitation, Remote medicine, COVID-19

\section{Background}

In the pandemic caused by SARS-CoV-2 infection, people worldwide are trying to prevent the spread of infection by practicing social distancing. Prolonged isolation at home leads to reduced physical activity in the general population, and the risks associated with physical inactivity are elevated, especially in patients with cardiac disease. However, now, many hospitals may not provide outpatient

\footnotetext{
* Correspondence: st7089-fki@umin.ac.jp; hmrt-tky@umin.ac.jp 'Department of Cardiovascular Medicine, Graduate School of Medicine, The University of Tokyo, 7-3-1 Hongo, Bunkyo-ku, Tokyo 113-8655, Japan Full list of author information is available at the end of the article
}

cardiac rehabilitation (CR) services in order to minimize the risk of SARS-CoV-2 infection in hospitals.

In Japan, the first coronavirus disease 2019 (COVID-19) case was reported on January 16, 2020; at that time, most hospitals continued outpatient CR. However, the incidence rate of infection gradually increased, and outpatient CR in Japan required close attention. During this period, we prohibited patients with common cold symptoms such as fever from participating in CR and encouraged all patients to wear masks in the exercise room. We conducted careful outpatient CR, disinfecting the ergometer handles and

(C) The Author(s). 2020 Open Access This article is licensed under a Creative Commons Attribution 4.0 International License, which permits use, sharing, adaptation, distribution and reproduction in any medium or format, as long as you give appropriate credit to the original author(s) and the source, provide a link to the Creative Commons licence, and indicate if changes were made. The images or other third party material in this article are included in the article's Creative Commons licence, unless indicated otherwise in a credit line to the material. If material is not included in the article's Creative Commons licence and your intended use is not permitted by statutory regulation or exceeds the permitted use, you will need to obtain permission directly from the copyright holder. To view a copy of this licence, visit http://creativecommons.org/licenses/by/4.0/. The Creative Commons Public Domain Dedication waiver (http://creativecommons.org/publicdomain/zero/1.0/) applies to the data made available in this article, unless otherwise stated in a credit line to the data. 
saddles with alcohol before and after each procedure. These prophylactic methods against COVID-19 for outpatient $\mathrm{CR}$ were recently recommended in the guideline prescribed by the European Association of Preventive Cardiology [1]. However, when a COVID19 case was reported in a sports gym, our hospital stopped providing outpatient CR services on March 4, 2020, a month ahead of the general hospitals since our hospital has a large heart transplantation center, with many patients suffering from severe heart failure (HF). The government declared a state of emergency on April 7 and asked the general public, the healthcare professionals, and medical contributors to refrain from unnecessary hospital visits. Due to this emergency declaration (ED), outpatient $\mathrm{CR}$ in most of the facilities has been interrupted. In addition, cardiologists in charge of outpatient CR also had to be involved in the treatment of SARS-CoV-2 infections, resulting in a greater mental and physical burden on them. Therefore, outpatient CR services were postponed.

The rate of readmission of patients with HF increases due to the inability to provide adequate medical care, including outpatient CR service [2]. What kind of CR can we provide in this COVID-19 era? Is it possible to provide safe and effective $\mathrm{CR}$ for patients with cardiac disease in their own houses? Here, we demonstrate the effectiveness of our remote CR program which had been launched in 2019, before the beginning of the COVID-19 pandemic.

\section{Methods}

Based on previous reports on successful tele-CR [3-5], we launched a home-based CR program for patients hospitalized for HF with a left ventricular ejection fraction (EF) of $<50 \%$, in January 2019. In the early phase of hospitalization, CR for patients with HF is initiated. CR for severe cases of HF (New York Heart Association [NYHA] class IV) is generally contraindicated. However, it is performed when disuse syndromes can potentially be prevented with careful bedside strength training. At discharge, patients could choose to participate in outpatient CR, remote CR, or non-CR. Outpatient CR was provided as a usual $\mathrm{CR}$ program according to the guideline by the Japan Circulation Society [6]. Patients who participated in remote $\mathrm{CR}$ were given digital video disk (DVD) guides for home CR, created by our CR staff; after discharge, a telephone consultation service was provided to the patients by cardiologists and specialized nurses, every 2 weeks for 5 months (Fig. 1). In our 15-min home CR film, the CR staff explained heart failure, warming-up exercise, aerobic exercises in and out of home, and possible symptoms requiring emergency visits. During the telephone consultations with the CR staff, patients were asked about the presence of leg edema, shortness of breath, and symptoms

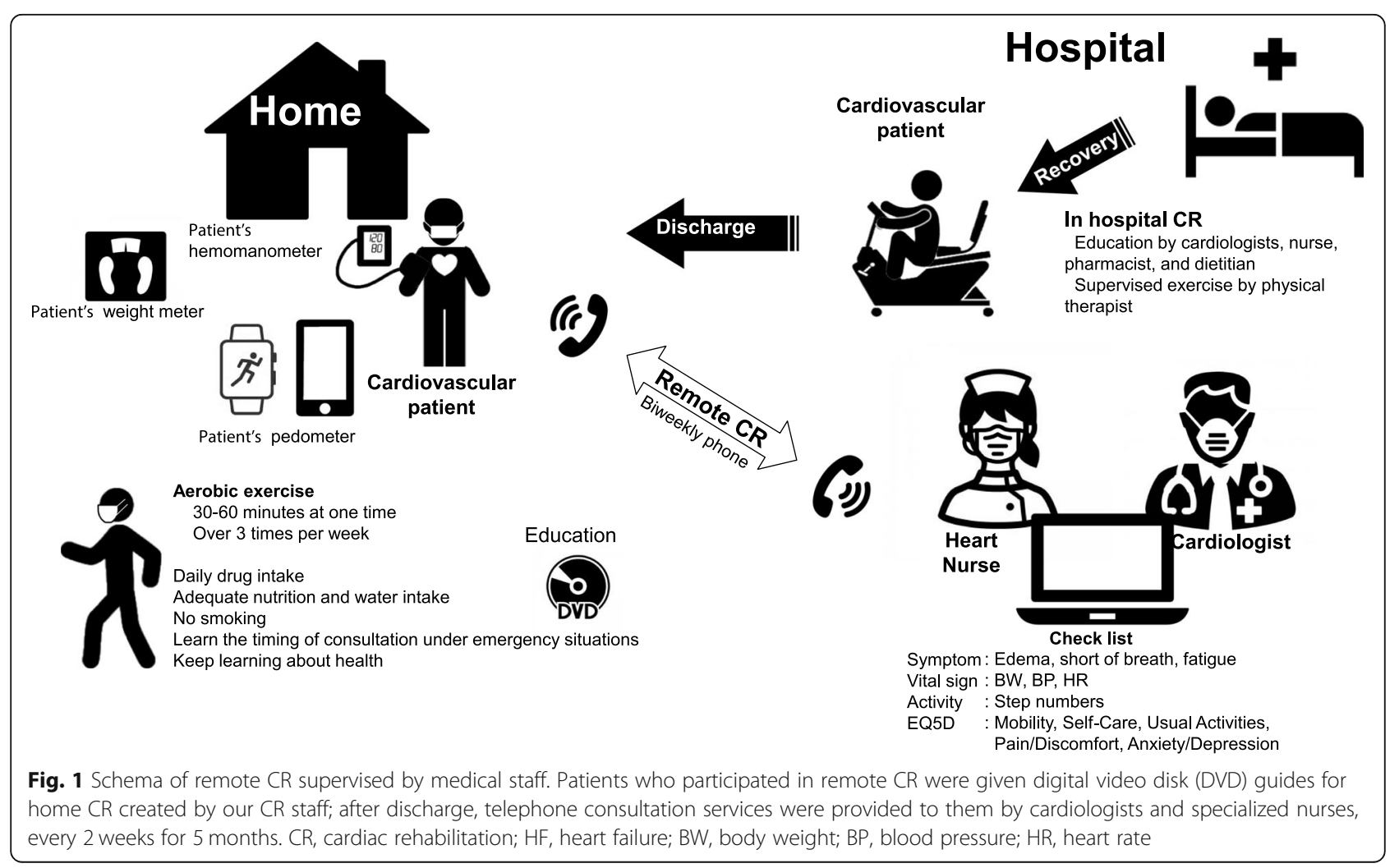


of fatigue along with body weight trends, blood pressure trends, pedometer trends, and questions on quality of life (QOL) based on EQ-5D [7].

Before outpatient CR closure in our hospital (on March 4, 2020), patients who were hospitalized for HF could choose whether to participate in outpatient CR, remote $\mathrm{CR}$, or non-CR at discharge (Fig. 2). To reduce the risk of SARS-CoV-2 infection, we promoted the remote CR option for HF patients. The patients who switched from the outpatient CR program to the remote CR program within 30 days of discharge were excluded, and emergency readmission rates within 30 days of discharge in patients with outpatient $C R$, remote $C R$, and non-CR were compared using the $\chi^{2}$ test. The EQ-5D scores in patients with outpatient $\mathrm{CR}$ and remote $\mathrm{CR}$ were compared using the unpaired $t$ test.

This study was based on the principles of the Declaration of Helsinki and approved by the ethical committees of the University of Tokyo Hospital, Tokyo, Japan (approval ID 2650-6).

\section{Results}

The number of participants in the remote CR program increased after the onset of SARS-CoV-2 infections; after March 4, 2020, our hospital stopped providing outpatient $\mathrm{CR}$ services, and instead, the remote $\mathrm{CR}$ service was provided after discharge. Accordingly, the proportion of the patients who initiated remote CR after discharge dramatically elevated from 19 to 69\% (Fig. 2). One patient who participated in the outpatient CR program and switched to the remote $\mathrm{CR}$ program within 30 days after discharge was excluded from the analysis. The dropout rate from the remote CR program was $0 \%$.

In a total of 236 patients hospitalized for HF (Table 1), there was a higher proportion of elderly and female patients in the remote $\mathrm{CR}$ and non-CR groups as compared to the outpatient CR group (70 \pm 9 years, $69 \pm 20$ years, and $59 \pm 18$ years, $P=0.01 ; 60 \%, 59 \%$, and $39 \%, P$ $=0.02$, respectively). The rate of emergency readmission within 30 days of discharge was lower in the CR groups, including the outpatient CR ( 2 out of $69,3 \%$ ) and remote CR (0 out of $30,0 \%)$ groups, as compared to the non-CR group (16 out of $137,12 \%)(P=0.02)$. The EQ$5 \mathrm{D}$ scores 30 days after discharge were higher in the remote $\mathrm{CR}$ group as compared to the outpatient $\mathrm{CR}$ group ( $0.87 \pm 0.15$ vs. $0.80 \pm 0.14, P=0.03)$, although the EQ$5 \mathrm{D}$ scores at discharge were similar for both groups.

\section{Discussion}

We could successfully demonstrate the improvement in the short-term emergency readmission rate in the remote $\mathrm{CR}$ and outpatient $\mathrm{CR}$ groups as compared to the non-CR group. The QOL score was rather high in the remote $\mathrm{CR}$ group as compared to the outpatient $\mathrm{CR}$ group. In Japan, as of May 15, 2020, the emergency readmission for cardiovascular diseases was not influenced by the COVID-19 pandemic. Therefore, we think that the effect of remote $\mathrm{CR}$ on emergency readmission could be accurately evaluated in this study.

In 2005, the first large study of remote CR for patients with HF $(n=426)$ was conducted in Europe to compare the clinical outcomes in three groups: the "nurse-telephone support" which was offered once a month, the

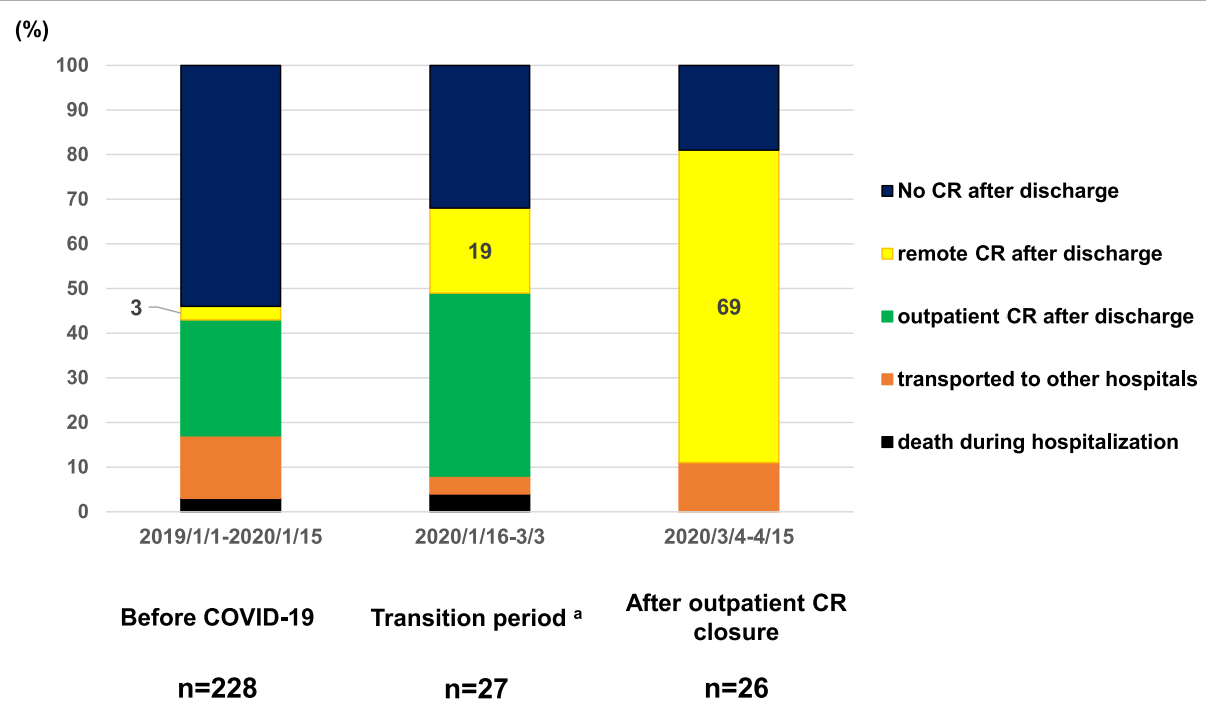

Fig. 2 Remote cardiac rehabilitation for patients with heart failure before and during the COVID-19 pandemic. CR after discharge for patients with HF before and during the COVID-19 pandemic. Superscript letter "a" denotes the period from the first report of COVID-19 in Japan to outpatient CR closure in our hospital. CR, cardiac rehabilitation 
Table 1 Clinical characteristics of patients hospitalized for HF

\begin{tabular}{|c|c|c|c|c|}
\hline & Outpatient CR & Remote $C R$ & non-CR & $\mathrm{P}$ \\
\hline $\mathrm{N}$ & 69 & 30 & 137 & \\
\hline \multicolumn{5}{|l|}{ Background } \\
\hline age, yrs. & $59 \pm 18$ & $70 \pm 9$ a & $69 \pm 20 b$ & .01 \\
\hline male $n, \%$ & $42(61 \%)$ & $12(40 \%)$ a & $56(41 \%) b$ & .02 \\
\hline $\mathrm{BMI}, \%$ & $25 \pm 6$ & $23 \pm 3$ & $24 \pm 6$ & .36 \\
\hline$E F, \%$ & $34 \pm 7$ & $33 \pm 10$ & $38 \pm 20$ & .11 \\
\hline NYHA IV n, \% & $8(12 \%)$ & $4(13 \%)$ & $18(13 \%)$ & .95 \\
\hline CAD n, \% & $9(13 \%)$ & $4(13 \%)$ & $18(13 \%)$ & 1.00 \\
\hline EQ5D score at discharge & $0.77 \pm 0.11$ & $0.82 \pm 0.19$ & - & .10 \\
\hline \multicolumn{5}{|l|}{ Outcome } \\
\hline EQ5D score 30 days after discharge & $0.80 \pm 0.14$ & $0.87 \pm 0.15 \mathrm{a}$ & - & .03 \\
\hline Emergency readmission $n, \%$ & $2(3 \%)$ & $0(0 \%)$ & $16(12 \%) b c$ & .02 \\
\hline
\end{tabular}

Patients' characteristics were compared using the $X^{2}$ test for non-continuous variables or an unpaired $t$ test for continuous variables

$C R$ cardiac rehabilitation, $H F$ heart failure, $B M I$ body mass index, EF ejection fraction, NYHA New York Heart Association, CAD coronary artery disease

${ }^{a} P<0.05$ between patients with outpatient $C R$ and remote $C R$

${ }^{\mathrm{b}} P<0.05$ between patients with outpatient $C R$ and non-CR

${ }^{c} P<0.05$ between patients with remote $C R$ and non-CR

"home telemonitoring and the nurse-telephone support" wherein the required medical equipment (weighing scale, sphygmomanometer, and electrocardiogram) was supplied to the patient and nurse telephone support was offered in the same manner as in the "nurse-telephone support" group, and the "usual care" by the primary care doctor [3]. The remote management groups showed a better cardiac prognosis than the "usual care" group. The "nurse-telephone support" and "home telemonitoring and nurse-telephone support" groups had the same levels of improved cardiac prognosis, suggesting the importance of nurse-telephone support by itself. In another large-scale remote CR study of patients with HF in the USA $(n=1437)$ [4], the remote CR group received telephone support by a nurse specialized in HF and medical device provision. As a result, the remote CR group had a better short-term prognosis than the standard treatment group. Recently, a large-scale remote CR study ( $n=$ 1571) was conducted among German patients hospitalized for HF [5]. In this study, patients were provided with medical devices including emergency liaison portable phones, physician-initiated 24-h medical support based on transmitted data, and monthly telephone support to provide HF education. As a result, cardiovascular events were significantly reduced in the remote CR group as compared to the standard care group (hazard ratio $0.70, P=0.028)$. According to these reports, telephone support by HF nurses and active intervention by physicians can be recognized as essential components for remote $\mathrm{CR}$ in patients with $\mathrm{HF}$. In addition, with telephone consultations by $\mathrm{CR}$ staff, the appropriate advice and reassurance could be provided to the patients, which is relevant to our findings that the EQ-5D scores 30 days after discharge were high in the remote CR group (Table 1). Concordantly, the dropout rate of the remote CR group was very low $(1-7 \%$ in the previous studies and $0 \%$ in our study), given that the reported dropout rate in outpatient CR ranged from 12 to $82 \%$ [8]. Although the outcomes of the remote CR group were compared only with those of the non-CR group in the previous reports [3-5], we could comprehensively compare the outcomes of outpatient $\mathrm{CR}$, remote $\mathrm{CR}$, and non-CR groups in this study.

The initiation of CR in the early phase of hospitalization followed by the patient's perception of heart failure appears to enable a smooth transition from in-hospital $C R$ to remote $C R$, reducing the medical staff's time and effort required for remote CR. In our hospital, the time burden of the medical staff that was managing the remote CR service was 180 min per month, consisting of weekly CR meetings and biweekly telephone consultations. Also, there were financial concerns associated with the introduction of remote CR. Basically, medical fees would be higher for remote $C R$. In addition, many telemedicine devices for patients with HF would be expensive. Our remote CR services amounted to, in total, approximately \$3000, including the initial expenses for the production of the video, DVD, and booklet, and telephone charges. Also, the medical devices were owned by each patient, and the number of steps per day was counted by the health app installed in each patient's mobile phone. Such financial burdens on the patients might affect their participation rate in the remote CR 
program. This should be resolved for the future promotion of remote CR.

Only 3.8\% of medical institutes in Japan could provide remote $C R$ as of April 13, 2020 [9]. Advancements in information technology and telemedicine equipment are expected to promote participation in the remote CR program. Large-scale studies using wearable devices such as Apple Watches have been conducted [10], but their low diagnostic accuracy makes it difficult to apply them into the remote CR of patients with HF. Remote monitoring of blood pressure, electrocardiogram, and oxygen saturation that is easily handled even by the elderly would enable more advanced remote $C R$. In addition, the problem of data security in remote management must be cleared [5]. Meanwhile, medical safety issues in remote management may also arise, which should also be addressed in order to promote remote $C R$ in the future [11]. The extent to which the healthcare supervisor is responsible for the clinical information detected on remote monitoring (e.g., ventricular tachycardia and ventricular fibrillation) can also pose a legal problem [12]. Each country's unique healthcare situation and relevant regulation system should be considered.

\section{Limitations}

Because our remote $C R$ program was launched in 2019 , the study population in the remote CR group is still small. However, during and after the COVID-19 pandemic, participation in the remote $C R$ program will be increasingly promoted. Further large-scale investigations on long-term prognosis of remote $C R$ are warranted. Considering that the main reason for nonparticipation in CR programs is the distance between patient residences and the hospital [13], the introduction of the remote $\mathrm{CR}$ program could possibly promote participation in CR. However, less-motivated patients might not participate even in remote CR program. At least, EQ-5D scores of anxiety and depression at discharge were not different between the outpatient $C R$ and remote $C R$ groups, which should be further investigated.

\section{Conclusions}

HF patients might be highly susceptible to COVID-19 infection. Therefore, cardiologists and nurses may be hesitant to commence CR for them. However, adequate management of heart diseases, including $C R$, is still clinically valuable. In order to improve the prognosis of HF patients, we can promote adequate lifestyle guidance and early CR intervention during hospitalization along with remote CR services with telephone support after discharge.

\section{Abbreviations}

CR: Cardiac rehabilitation; HF: Heart failure; EF: Ejection fraction;

ED: Emergency declaration; NYHA: New York Heart Association; DVDs: Digital video disks; QOL: Quality of life

\section{Acknowledgements}

We thank Yuto Konishi, Masanobu Taya, Hidetaka Itou, Takao Kato, Akira Nakamura, and Eri Nakamura for making an educational movie regarding remote $C R$.

\section{Authors' contributions}

Conceptualization: AN. Data curation: AN, NT, MK, KH, NM, and TF. Formal analysis: AN and NT. Funding acquisition: AN. Investigation: AN and NT. Methodology: AN and HM. Project administration: AN. Resources: NM. Supervision: IK. Writing, review, and editing: AN and HM. All authors read and approved the final manuscript.

\section{Funding}

This report was supported by the Yuumi Memorial Foundation for Home Health Care.

\section{Availability of data and materials}

The datasets used and analyzed in the presented study are available from the corresponding author on reasonable request.

\section{Ethics approval and consent to participate}

The study was based on the principles of the Declaration of Helsinki and approved by the ethical committees of the University of Tokyo Hospital, Tokyo, Japan (approval ID 2650-6).

Consent for publication

Not applicable.

\section{Competing interests}

The authors declare that they have no competing interests.

\section{Author details}

${ }^{1}$ Department of Cardiovascular Medicine, Graduate School of Medicine, The University of Tokyo, 7-3-1 Hongo, Bunkyo-ku, Tokyo 113-8655, Japan.

${ }^{2}$ Nursing Department, The University of Tokyo, Tokyo, Japan.

Received: 20 July 2020 Accepted: 20 August 2020

Published online: 05 September 2020

\section{References}

1. Recommendations on how to provide cardiac rehabilitation activities during the COVID-19 pandemic. Released by Secondary Prevention and Rehabilitation Section of the European Association of Preventive Cardiology (EAPC) Update: 7 April 2020.

2. Lippi G, Henry BM, Sanchis-Gomar F. Physical inactivity and cardiovascular disease at the time of coronavirus disease 2019 (COVID-19). Eur J Prev Cardiol. 2020 Apr;9:2047487320916823. https://doi.org/10.1177/2047487320916823.

3. Cleland JG, Louis AA, Rigby AS, Janssens U, Balk AH. TEN-HMS Investigators. Noninvasive home telemonitoring for patients with heart failure at high risk of recurrent admission and death: the TransEuropean Network-Home-Care Management System (TEN-HMS) study. J Am Coll Cardiol. 2005;45(10):1654-64.

4. Ong MK, Romano PS, Edgington S, Aronow HU, Auerbach AD, Black JT, et al. Better Effectiveness After Transition-Heart Failure (BEAT-HF) Research Group. Effectiveness of remote patient monitoring after discharge of hospitalized patients with heart failure: the Better Effectiveness After Transition -- Heart Failure (BEAT-HF) Randomized Clinical Trial. JAMA Intern Med. 2016;176(3):310-8. https://doi.org/10.1001/jamainternmed.2015.7712.

5. Koehler F, Koehler K, Deckwart O, Prescher S, Wegscheider K, Kirwan BA, et al. Efficacy of telemedical interventional management in patients with heart failure (TIM-HF2): a randomised, controlled, parallel-group, unmasked trial. Lancet. 2018;392(10152):1047-57. https://doi.org/10.1016/S0140-6736(18)31880-4.

6. JCS Joint Working Group. Guidelines for rehabilitation in patients with cardiovascular disease (JCS 2012). Circ J. 2014;78(8):2022-93.

7. Van Spall HGC, Lee SF, Xie F, Oz UE, Perez R, Mitoff PR, et al. Effect of patient-centered transitional care services on clinical outcomes in patients 
hospitalized for heart failure: the PACT-HF Randomized Clinical Trial. JAMA. 2019;321 (8):753-61. https://doi.org/10.1001/jama.2019.0710.

8. Turk-Adawi Kl, Grace SL. Narrative review comparing the benefits of and participation in cardiac rehabilitation in high-, middle- and low-income countries. Heart Lung Circ. 2015;24(5):510-20. https://doi.org/10.1016/j.hlc. 2014.11.013.

9. The report of the cardiac rehabilitation during COVID-19. Data by the Japanese Association of Cardiac Rehabilitation. http://www.jacr.jp/web/ wp-content/uploads/2020/05/COVID-19questionnaire.pdf. (Accessed 2 June, 2020).

10. Seshadri DR, Bittel B, Browsky D, Houghtaling P, Drummond CK, Desai MY, et al. Accuracy of Apple Watch for detection of atrial fibrillation. Circulation. 2020;141(8):702-3. https://doi.org/10.1161/CIRCULATIONAHA.119.044126.

11. Jamil F, Ahmad S, lqbal N, Kim DH. Towards a remote monitoring of patient vital signs based on loT-based blockchain integrity management platforms in smart hospitals. Sensors (Basel). 2020;20. pii: E2195. doi: 10.3390/s20082195.

12. Piotrowicz E, Piepoli MF, Jaarsma T, Lambrinou E, Coats AJ, Schmid JP, et al. Telerehabilitation in heart failure patients: the evidence and the pitfalls. Int J Cardiol. 2016;220:408-13. https://doi.org/10.1016/j.jicard.2016.06.277.

13. Nakayama A, Nagayama M, Morita H, Tajima M, Mahara K, Uemura Y, et al. A large-scale cohort study of long-term cardiac rehabilitation: a prospective cross-sectional study. Int J Cardiol. 2020;309:1-7. https://doi.org/10.1016/j. ijcard.2020.03.022.

\section{Publisher's Note}

Springer Nature remains neutral with regard to jurisdictional claims in published maps and institutional affiliations.

Ready to submit your research? Choose BMC and benefit from:

- fast, convenient online submission

- thorough peer review by experienced researchers in your field

- rapid publication on acceptance

- support for research data, including large and complex data types

- gold Open Access which fosters wider collaboration and increased citations

- maximum visibility for your research: over $100 \mathrm{M}$ website views per year

At $\mathrm{BMC}$, research is always in progress.

Learn more biomedcentral.com/submissions 